\title{
Anxiety and Depression Scores in Maternity Healthcare Workers during the Covid-19 Pandemic
}

\author{
Gillian Corbett ${ }^{1}$, Sarah Milne ${ }^{1}$, Shuja Reagu ${ }^{2}$, Suruchi Mohan ${ }^{3}$, Mark Hehir ${ }^{4}$, Stephen \\ Lindow $^{1}$, and Michael O Connell ${ }^{1}$ \\ ${ }^{1}$ Coombe Women and Infants University Hospital \\ ${ }^{2}$ Hamad Medical Corporation \\ ${ }^{3}$ Weill Cornell Medical College in Qatar \\ ${ }^{4}$ Columbia University, College of Physicians and Surgeons
}

May 20, 2020

Anxiety and Depression Scores in Maternity Healthcare Workers during the Covid-19 PandemicGillian A. Corbett $^{1}$, Sarah J. Milne ${ }^{1}$, Suruchi Mohan ${ }^{2}$, Shuja Reagu ${ }^{3}$, Stephen W. Lindow ${ }^{1}$, Mark P. Hehir ${ }^{1}$, Michael P O'connell ${ }^{1}$.

\begin{abstract}
Affiliations
${ }^{1}$ Department of Obstetrics and Gynaecology, Coombe Women and Infants University Hospital, Cork Street, Merchants Quay, Dublin 8, Ireland. D08 XW7X² Sidra Medicine, Doha, Qatar ${ }^{3}$ Hamad Medical Corporation, Doha, Qatar
\end{abstract}

\section{Corresponding Author}

Dr Gillian Corbett.

Department of Obstetrics and Gynaecology, Coombe Women and Infants University Hospital, Cork Street, Merchants Quay, Dublin 8, Ireland. D08 XW7X Tel: +353 (1) 4085200

Email: gcorbett@coombe.ie

Word Count: 598

Financial Disclaimers/Conflict of Interest: None

\section{INTRODUCTION}

Severe-Acute-Respiratory-Syndrome-CoronaVirus-2 (SARSCoV-2/Covid-19) increases morbidity in healthcare workers (HCWs), who make up a significant proportion of infections, hospital admissions and deaths ${ }^{1}$. Aside from physical illness, covid-19 significantly affects psychological health in the general population, in pregnant women ${ }^{2}$ and HCWs. Working on the frontline compounds the psychological burden on providers caring for patients with Covid-19 $9^{3-4}$. It is essential that healthcare institutions understand the stress their staff are experiencing, which may translate into anxiety and depressive symptomatology. To this end we assessed anxiety and depression among HCWs due to the Covid-19 pandemic.

$M E T H O D S$ 
A prospective study was performed on HCWs at a tertiary-level university hospital in Dublin, Ireland. The Coombe Women and Infants University Hospital (CWIUH) is one of the largest providers of women's healthcare in Europe, delivering almost 9000 babies annually and serving the diverse urban population of this European capital city.

The anonymous online survey was distributed to HCWs in all departments of the hospital.

The Generalized-Anxiety-Disorder-7(GAD-7 $)^{5}$ and Patient-Health-Questionnaire-9(PHQ-9 ${ }^{6}$ scores were used to analyze severity of anxiety and depression (A\&D). The composite questionnaire included questions assessing previous mental health status, coping strategies and support mechanisms.

Survey responses were analyzed using statistical software (IBM-SPSS-Version-24.0). Median and range PHQ-9 and GAD-7 scores were compared using Mann-Whitney-U or Kruskal-Wallis-H tests. Due to rapid emergence of the pandemic, the study had executive ethical approval.

\section{RESULTS}

From $20^{\text {th }}$ April to $5^{\text {th }}$ May 2020, 252 hospital staff completed the questionnaire. Nursing (35\%) and administrative staff (22\%) made up the largest proportion of the cohort followed by medical staff(15\%).

HCW mental health has significantly deteriorated since the onset of Covid-19. 46.6\% reporting their mood has worsened. HCWs reported significant levels of A\&D with $20.3 \%$ having moderate to severe depression and $21.0 \%$ having moderate to severe anxiety(Table 1). A\&D scores significantly varies with age(Table 2), with higher scores in younger age groups(GAD-7 9.00vs1.00; $\mathrm{p}=0.000$, PHQ-9 9.00.vs.0.5; $=0.0001$ ). Anxiety scores were higher in women but depression scores were similar between genders (GAD-7 5.00vs.2.00; $\mathrm{p}=0.027$; PHQ-9 4.00vs.3.00; $\mathrm{p}=0.144)$. A\&D scores were higher amongst administrative staff than medical staff(GAD-7 7.00vs.3.00,p=0.001; PHQ-9 6.00vs.2.00,p=0.010)(Table 2).

When questioned on social isolation, 44\%(112/252) of staff reported loneliness, 35\%(88/252) felt low due to limitation of activities, $44 \%(110 / 252)$ felt low from being separated from their families. Tensions at home were reported by healthcare workers, with $13.5 \%(34 / 252)$ reporting tensions with their partner and $19 \%(48 / 252)$ reporting tensions with other household members including children.

\section{DISCUSSION}

While high rates of physical morbidity from Covid-19 amongst HCWs have been demonstrated, examining the psychological effects of Covid-19 of HCWs is critical.

Significant proportions of staff are experiencing a deterioration in their mental health due to Covid-19, with over $20 \%$ having moderate to severe depression and $20 \%$ with moderate to severe anxiety.

Subgroup analysis revealed variations in psychological distress. A\&D scores were higher among administrative staff when compared with front-line medical staff, with higher anxieties scores in female HCWs. Despite younger people having better prognosis than their older counterparts, scores were significantly higher in younger HCWs. Older colleagues may have more matured coping mechanisms or younger HCWs may be worrying about their older relatives, as this group saw in a similar study on pregnant women ${ }^{2}$. Increased levels of A\&D in administrative staff compared to front line health care providers may represent a rationalization of risk in doctors and nurses/midwives as part of their professional training.

While HCWs care for patients during this crisis, we must recognize that HCWs are not immune to significant psychological distress. As the pandemic progresses, the psychological burden is likely to worsen. Recognizing this can aid healthcare leaders to support HCWs, prevent burnout and retain their confidence as we deal with the ongoing pandemic.

\section{Author contributorship:}

The study concept was conceived by MPOC and SWL. Study was designed by SWL MPH, SM, SR and GAC. Data collection was performed by GAC and SJM, and was analysed by GAC. The manuscript was 
drafted by GAC and edited by SJM, SM, SR, MPH, SWL and MPOC.

\section{Conflict of Interest:}

The authors have no conflicts of interest to disclose.

\section{Financial Disclaimers:}

None.

\section{Ethics approval:}

Suspension of institutional research ethics committee meetings due to the Covid-19 outbreak meant that approval for the study was granted on $8^{\text {th }}$ April 2020 by the Coombe Women and Infants University Hospital clinical governance board and executive committee.

\section{References}

Health Do. Latest Updates on COVID-19 (Coronavirus). In. gov.ie25th April 2020..

Corbett GA, Milne SJ, Hehir MP, Lindow SW, O'Connell MP. Health anxiety and behavioural changes of pregnant women during the COVID-19 pandemic.Eur J Obstet Gynecol Reprod Biol. 2020.

Lai J, Ma S, Wang Y et al. Factors Associated With Mental Health Outcomes Among Health Care Workers Exposed to Coronavirus Disease 2019. JAMA Netw Open 2020 Mar 2;3(3):e203976.

Wang C, Pan R, Wan X, et al. Immediate Psychological Responses and Associated Factors during the Initial Stage of the 2019 Coronavirus Disease (COVID-19) Epidemic among the General Population in China.Int $J$ Environ Res Public Health. 2020;17(5).

Spitzer RL, Kroenke K, Williams JB, Löwe B. A brief measure for assessing generalized anxiety disorder: the GAD-7. Arch Intern Med. 2006;166(10):1092-1097.

Kroenke K, Spitzer RL, Williams JB. The PHQ-9: validity of a brief depression severity measure. J Gen Intern Med.2001;16(9):606-613.

Table caption list.

Table 1. Healthcare workers participant demographics and health anxieties influential factors

Table 2: Variation in GAD-7 and PHQ-9 scores with baseline characteristics

\section{Hosted file}

Table 1.docx available at https://authorea.com/users/324567/articles/452736-anxiety-anddepression-scores-in-maternity-healthcare-workers-during-the-covid-19-pandemic

\section{Hosted file}

Table 2.docx available at https://authorea.com/users/324567/articles/452736-anxiety-anddepression-scores-in-maternity-healthcare-workers-during-the-covid-19-pandemic 